\title{
The Co-Regulation of the Gut Microbiome and Host Genes Might Play Important Roles in Metformin Intolerance
}

\section{Huixiang Zhang}

Kunming Medical University

Jiao Lai

Qujing First People's Hospital: Kunming Medical University Affiliated Qujing Hospital

Lihuan Zhang

Kunming Medical University

Wei Zhang

Qujing First People's Hospital: Kunming Medical University Affiliated Qujing Hospital

Xun Liu

Kunming Medical University

Qilin Gong

Qujing First People's Hospital: Kunming Medical University Affiliated Qujing Hospital

\section{Rui Zhao}

Qujing First People's Hospital: Kunming Medical University Affiliated Qujing Hospital

\section{Dongqing Li}

Qujing First People's Hospital: Kunming Medical University Affiliated Qujing Hospital

\section{Hehua Huang}

Qujing First People's Hospital: Kunming Medical University Affiliated Qujing Hospital

\section{Ya Zhao}

Qujing First People's Hospital: Kunming Medical University Affiliated Qujing Hospital

\section{Shan Yan}

Kunming Medical University

\section{Ming Yu}

Kunming Medical University

Xiaodan Liu

Yunnan Customs District P.R. China

\section{Lanlan Shi}

Kunming Medical University

\section{Limei Wang}

Kunming Medical University

\section{Weiwen Chen}


Qujing First People's Hospital: Kunming Medical University Affiliated Qujing Hospital

\section{Xue Cao ( $\nabla$ dir1865@163.com)}

Kunming Medical University https://orcid.org/0000-0002-1303-9604

\section{Research}

Keywords: Type 2 diabetes, Metformin, Gastrointestinal intolerance, Co-regulation, Gut microbiome, Host genes

Posted Date: October 20th, 2021

DOl: https://doi.org/10.21203/rs.3.rs-965098/v1

License: (9) This work is licensed under a Creative Commons Attribution 4.0 International License. Read Full License 


\section{Abstract \\ Background}

Metformin is commonly considered the first-line therapy for type 2 diabetes (T2D) and also had potential treating utility in other areas; however, $\sim 20 \%$ of patients experience intolerance with unclear underlying mechanisms. In the present study, we performed the full-length 16S rRNA (V1-V9) for the fecal samples and bioinformatics analysis to study the mechanisms of the metformin intolerance combining the gut flora and host.

\section{Results}

The results showed that Barnesiella $(\mathrm{p}=0.046)$ and Parabacteroides goldsteinii $(\mathrm{p}=0.016)$, which transforming primary into secondary bile acid (SBA), were higher in the TS than T group, and were eliminated in the TSa group, which might lead to the accumulation of primary bile acids (PBA) such as cholic acid (CA), the change of GL/1 gene, and following diarrhea in the TSa group. Lactobacillus brevis $(\mathrm{p}=0.024)$ and Lactobacillus plantarum $(\mathrm{p}=0.026)$ were up-regulated in TSa than TS group. The two flora might cause the changes of genes including FOXA2, HTR7, GADPH, and intolerance relief, which might be a worthwhile future direction for preventing metformin intolerance.

\section{Conclusions}

These results hinted that the differential flora and co-regulation of them with the host might be intolerance-related. Our results partly provided theoretical support for intolerance prevention.

\section{Background}

Metformin is commonly considered as the first-line therapy for type 2 diabetes (T2D) and also had potential treating utility in obesity, metabolic dysfunction, and some cancers. However, $\sim 20 \%$ of patients experience gastrointestinal (GI) intolerance, including diarrhea, nausea, and bloating with unclear underlying mechanisms and a lack of effective management strategies that warrant discontinuation of metformin treatment [1]. Researches have uncovered that the modification of intestinal microbiota and host DNA methylation might be both involved in GI intolerance, and the increase of Escherichia abundance might be one of the reasons in the European population. However, the Escherichia have not been modified in the Chinese population [2], which implied that different mechanisms might employed in Chinese.

In present study, we collected fecal samples from T2D patients who had no GI intolerance after metformin administration (before $(\mathrm{T})$ and after ( $\mathrm{Ta}$ ) taking metformin, respectively) and who had $\mathrm{GI}$ intolerance after metformin administration (before (TS) and after (TSa) taking metformin, respectively) 
and health subjects $(\mathrm{N})$. Then, we carried out 16S rRNA sequencing on fecal samples and bioinformatics analysis to explore the mechanisms of metformin intolerance.

\section{Results And Discussion}

We found that the Dorea longicatena was up-regulated in both T and TS groups (LDA Score>2.0) and also in the merging data than $\mathrm{N}$ group (Figure S 1a-c). The K03386, K01809, K03321, and K13016 are enriched in the T group, and $\mathrm{K} 02398$ is enriched in the $\mathrm{N}$ group (Table 1). The alpha diversity was not differed before vs. after taking metformin (Table S2). These patterns had been confirmed by previous studies [3, 4], which proved the high confidence of our research.

Table 1

Partial KOs significant difference between groups

\begin{tabular}{|c|c|c|}
\hline Group & KO Class & KO ID and Definition \\
\hline \multirow[t]{5}{*}{$\mathrm{T}-\mathrm{N}$} & \multirow[t]{4}{*}{ KOs up-regulated in T group } & $\begin{array}{l}\text { K03386: PRDX2_4, ahpC; peroxiredoxin (alkyl } \\
\text { hydroperoxide reductase subunit C) [EC:1.11.1.15] }\end{array}$ \\
\hline & & $\begin{array}{l}\text { K01809: manA, MPI; mannose-6-phosphate } \\
\text { isomerase [EC:5.3.1.8] }\end{array}$ \\
\hline & & K03321: TC.SULP; sulfate permease, SulP family \\
\hline & & $\begin{array}{l}\text { K13016: wbpB; UDP-N-acetyl-2-amino-2- } \\
\text { deoxyglucuronate dehydrogenase [EC:1.1.1.335] }\end{array}$ \\
\hline & KOs up-regulated in $\mathrm{N}$ group & $\begin{array}{l}\text { K02398: flgM; negative regulator of flagellin } \\
\text { synthesis FlgM }\end{array}$ \\
\hline \multirow[t]{6}{*}{ T-TS } & \multirow{6}{*}{$\begin{array}{l}\text { Flagellin-related KOs up-regulated in } \\
\text { TS groups }\end{array}$} & K02409: fliF; flagellar M-ring protein \\
\hline & & $\begin{array}{l}\text { K02389: flgD; flagellar basal-body rod modification } \\
\text { protein }\end{array}$ \\
\hline & & K02397: flgL; flagellar hook-associated protein 3 \\
\hline & & K02413: fliJ; flagellar FliJ protein \\
\hline & & $\begin{array}{l}\text { K02398: flgM; negative regulator of flagellin } \\
\text { synthesis }\end{array}$ \\
\hline & & K02395: flgJ; flagellar protein \\
\hline $\begin{array}{l}\text { TS- } \\
\text { TSa }\end{array}$ & $\begin{array}{l}\text { Lactose degradation related KO } \\
\text { down-regulated in TSa than TS } \\
\text { group }\end{array}$ & K01190: lacZ; beta-galactosidase [EC:3.2.1.23] \\
\hline $\mathrm{T}-\mathrm{Ta}$ & $\begin{array}{l}\text { RFO degradation related KO up- } \\
\text { regulated in Ta than T groups }\end{array}$ & K07406: melA; alpha-galactosidase [EC:3.2.1.22] \\
\hline
\end{tabular}

Importantly, we found that the background difference and changes caused by metformin in the gut microbiome might be both involved in GI intolerance. The results showed that the Barnesiella $(p=0.046)$ 
and Parabacteroides goldsteinii $(\mathrm{p}=0.016)$, which transforming primary into secondary bile acids (SBA) $[5,6]$ were far higher in the TS than T group. However, the two flora were eliminated in the TSa group (Figure 1a), which implied that the eradication of Barnesiella and $P$. goldsteinii might lead to the accumulation of PBA such as CA and following diarrhea in the TSa group (Figure 2 inside (1) dashed rectangle). Six flagella assembly-relevant Kyoto Encyclopedia of Genes and Genomes (KEGG) orthologs (KOs) significantly higher enriched in TS than T group were also found (Table 1), which might cause a higher risk of inflammation in the TS group for the pro-inflammatory potential of flagellin [7]. The TS and T groups exhibited different patterns of microbial communities from TSa and Ta groups (Figure 1b). There are 26 and 10 significantly differential flora in total between the TS and TSa group, and the T and Ta group, respectively (Figure $1 \mathrm{c}$, Table $\mathrm{S} 2,3)$. Among them, Leuconostocaceae $(\mathrm{p}=0.048)$, Leuconostoc $(\mathrm{p}=0.041)$, Weissella confusa $(\mathrm{p}=0.037)$, Weissella paramesenteroides $(\mathrm{p}=0.008)$, Lactobacillus brevis $(p=0.024)$, Lactobacillus plantarum $(p=0.026)$ were up-regulated in TSa group (Figure $1 d)$. These flora belongs to lactic acid bacteria. In addition, the alpha-galactosidase, a protective factor of GI intolerance [8], was higher enriched in Ta than T group but not in the TSa group. In contrast, beta-galactosidase, also a protective factor of GI intolerance [9], was down-regulated in TSa compared with the TS group (Table 1). It may be one reason for $\mathrm{Gl}$ intolerance (Figure 2, represented by black and pink lines respectively inside (2) dashed ellipse).

Besides, by bioinformatics analysis as that showed in Supplementary, we found that metformin intolerance might be the result of co-regulation of intestinal flora and host genes. There is a closeinteraction among the L. plantarum, L. brevis, cholic acid (CA) and, metformin glycemic response and intolerance-related genes (Figure 1e, Table S4-6). According to GEO data and previous reports, we found that the CA and FOXA2 could both modulate the expression of GL/1 [10]. In contrast, the L. plantarum and L. brevis could respectively modulate the expression of HTR7 and GAPDH. By RT-qPCR check, we found the expression of GLI1 was down-regulated $(\mathrm{p}<0.05)$, and the expression of FOXA2 showed an upward trend $(p<0.10)$ in TSa than TS group (Figure 2, Figure S3, Table S7). The downregulation of GL/1 might cause bloating, diarrhea and nausea by sharing the same pathogenesis and symptoms of inflammatory bowel disease (IBD), which might lead to GI intolerance [11]. On the contrary, we found expression of HTR7 and GADPH showed a downward trend $(\mathrm{p}<0.10)$ in TSa than TS group (Figure 2, Figure S3, Table S7), which might be related to the relief of GI intolerance, for opposite changes of these genes are corresponding to IBD $[12,13]$. The effect of flora on these genes seem to restore a healthy state after being disturbed, which might be one of the reasons why some intolerance individuals become more tolerant to metformin after persisting on it for a period, which might be a worthwhile future direction for the prevention of metformin GI intolerance.

\section{Conclusion}

In general, the mechanism of metformin intolerance in Chinese might be different from that in Europeans. It might be caused by the eradication of PBA degrading-related flora in gut microbiota and also the coregulation of intestinal flora and host genes. However, the relatively small sample size might affect the results to a certain extent. However, fortunately, some of the results in our study are consistent with large 
cohort researches, which guaranteeing the accuracy of the results. More importantly, the selection of dose regimen and treatment duration was based on the hospital's conventional treatment process to avoid interference treatment and better reflect the actual clinical phenomenon. Therefore, our results could still provide theoretical support for intolerance prevention. Further experiments with larger sample sizes and animal models are required to verify those results to reveal the mechanism involved so that more people could benefit from metformin in the future.

\section{Material And Methods}

\section{Subjects}

This study was carried out at the First People's Hospital of Qujing City, Yunnan Province『China, from February 2019 to August 2019. The inclusion criteria were as follows: the patients were newly diagnosed with T2D with the fasting plasma (blood) glucose higher than $7.0 \mathrm{mmol} / \mathrm{L}$, were between 40 to 70 years old, were able to communicate, had volunteered to participate in this study, and were willing to provide informed consent. Subjects did not take ion pump inhibitor drugs, antibiotics, steroid hormones, or Chinese herbal medicine, including oral, intramuscular, or intravenous injections within the three months before collecting fecal samples, did not take other glucose-lowering medications. Subjects did not take other medicine except drugs used in this study during the experiment, did not occur diarrhea on the day of the first sampling. Those excluded were the patients who had severe conditions, including indigestion, renal failure, hepatic failure, severe gallbladder, stroke, pancreatic diseases, malignant tumors, or unstable cardiovascular diseases (such as myocardial infarction, ketosis, or hyperthyroidism) [14]. Age-matched healthy volunteers were included as above.

\section{Medication Strategy and Samples Collection}

We recommend the use of an oral glucose tolerance test (OGTT) (consisting of a fasting and 2-hour glucose level using a 75-g oral glucose load) to screen for impaired glucose tolerance (IGT) and T2D. Feces and blood samples were collected, and the OGTT experiment was completed in the early morning of the next day after the patient was admitted to the hospital. The blood was used to detect other indicators such as fasting blood glucose, serum C-peptide. Then the patient took metformin hydrochloride sustained-release tablet (Qingdao Huanghai Pharmaceutical Co., Ltd.) orally at a dose of $500 \mathrm{mg} /$ time, two times/day. When the patient had intestinal side effects, they stopped metformin treatment, collected stool, and measured fasting blood glucose the next morning. When the patient had no side effects after metformin administration, the stool was collected five days later, and fasting blood glucose was measured. The feces of each subject were immediately stored at $-80^{\circ} \mathrm{C}$ after collection until the next step. According to hospital clinical experience, insulin combined with metformin treatment can achieve a better hypoglycemic effect, and insulin will not change the composition of intestinal flora [2]. So the patient had been treated with an insulin pump with the weight $(\mathrm{kg}) * 0.2-0.5 \mathrm{u} /$ day dose first for a day, when whose random blood glucose was greater than $16.8 \mathrm{mmol} / \mathrm{L}$ on admission. Feces and blood samples were collected from the healthy subjects only one time, respectively. 


\section{Isolation and qualification of fecal bacterial DNA}

Eighteen stool samples were collected from twelve subjects (Table S1), as follows: six samples from three patients who had no intestinal side effects after metformin administration (before $(T)$ and after (Ta) taking metformin, respectively). Six samples from three patients who had intestinal side effects after metformin administration (before (TS) and after (TSa) taking metformin, respectively). Six samples from six health subjects $(\mathrm{N})$. Genomic DNA from human stool samples clinically collected was extracted by a modified CTAB method [15]. DNA concentration, purity was monitored and was diluted to proper concentration.

\section{PCR amplification of 16S rRNA V1-V9 and high-throughput sequencing}

The full V1-V9 region of the bacterial 16S rRNA gene was amplified using the universal primer set 27F and 1492R with Barcode by using third-generation sequencing [16]. The PCR products were mixed and purified. The sequencing library was generated, assessed, and sequenced on the PacBio Sequel platform using standard protocols [17].

\section{Processing of sequencing data}

The original sequences were registered in the NCBI SRA database (registration number: PRJNA725340). The clean reads were acquired by removing the barcodes and primers, low-quality reads, and chimera sequences from raw data $[18,19]$. Sequences with $\geq 97 \%$ similarity were assigned to the same OTUs by Uparse software (Uparse v7.0.1001) [20]. The representative sequence for each OTU was screened for further annotation. The taxonomic information for each representative sequence was annotated by the SSUrRNA Database [21] of Silva Database [22] based on the Mothur algorithm. Alpha diversity such as Chao1, Shannon index were calculated with QIIME (Version1.9.1) and displayed with R software (Version 2.15.3). The Chao1 index was selected to identify community richness, and the Shannon index was used to identify community diversity. Tukey and Wilcox's tests were used to analyze the differences between groups $\llbracket p<0.05$ was considered statistically significant. Principal Coordinate Analysis (PCoA) based on unweighted unifrac distance calculated by QIIME software (Version 1.9.1) and displayed by WGCNA package, stat packages, and ggplot2 package in R software (Version 2.15.3). The significantly bacterial taxa between groups were identified by the linear discriminate analysis (LDA) effect size (LEfSe) method with a LDA threshold value of $2.0^{18}$ and MetaStats at $95 \%$ confidence interval, simultaneously. Potential functional contributions of the observed microbes were inferred using PICRUSt2 [23]. Significantly different Kyoto Encyclopedia of Genes and Genomes (KEGG) orthologs (KOs) were identified by t-test, P $<0.05$ was considered statistically significant. The corresponding enrichment pathways of significantly different KOs were predicted in KEGG囚https://www.kegg.jp/kegg/tool/map_pathway2.html】.

\section{Bioinformatics analysis}

In order to explore the effects of intolerance-related differential bacteria and primary bile acid on the body, we used the GEO database to search the research on them. As a result, we found the GSE23630 data set 
related to Lactobacillus plantarum 299v囚the GSE41734 data related to Lactobacillus brevis 119-2, the GSE55443 data related to cholic acid. Besides, Using related scores $>1.0$ as the cutoff $₫$ we searched metformin intolerance symptoms, including bloating, diarrhea, and nausea in GeneCards (https://www.genecards.org/) to collect intolerance symptoms related genes.

Next, we used GEO2R to analyze the above two data sets related to the differential bacteria and carry out the T-test separately. Using $\mathrm{P}<0.05$ as the cutoff, genes significantly different between the phorbol 12 myristate 13-acetate (PMA)/ionomycin (IO)-induced intestinal explants pro-inflammatory disease model and Lactobacillus plantarum 299v treated samples, and between livers samples from Lactobacillus brevis 119-2 and control diet-administrated rat were filtered out. If there are multiple transcripts for the same gene, we multiplied the P-value and used the square root of the product as the final P-value. Because there is only 1 sample in the case and control groups in the GSE55443 data set, we could not carry out statistical analysis. So, we selected the top 100 different genes with the maximum value and the top 100 genes with the minimum value of LogFC between CA and vehicle-treated intestinal epithelial cells of mice. Then, we intersected the differential genes obtained from the above analysis with the genes related to intolerance symptoms.

After that, Protein interaction analysis on differential genes from the above analysis, and seven genes related to metformin glycemic response, and four genes related to metformin intolerance retrieved from the literature [24] was performed by String (https://string-db.org/) [25]. The interaction results were visualized by Cytoscape [26].

\section{RNA Isolation and cDNA Synthesis}

Total RNA was isolated from a 1ml whole blood sample with Trizol (Invitrogen, USA) reagent and purified using RNA simple Total RNA Kit (TIANGEN, China) followed the manufacturer's instructions. About $0.2 \mu \mathrm{g}$ of total RNA was used for first-strand cDNA synthesis by using Mix in FastKing RT Kit (With gDNase) (TIANGEN, China) according to the manufacturer's instructions.

\section{Primer Design and Evaluation}

The primer pairs of FOXA2, GLI1, HTR7, and GAPDH were designed according to their sequences by using the online program Primer-BLAST (Table S9) (https://www.ncbi.nlm.nih.gov/tools/primer-blast/ and https://pga.mgh.harvard.edu/primerbank/index.html). The primer evaluation was carried out according to the reported method of literature [27] with modification. Standard curves of each primer pair were established using 8-fold dilution series of template cDNA.

\section{Real-Time PCR}

The quantitative real-time PCR was performed using FastStart Essential DNA Green Master (Roche, Switzerland) with a Real-Time PCR System (BIO-RAD, USA). Each reaction contained $5 \mu \mathrm{L}$ cDNA (8-time diluted), $10 \mu \mathrm{L}$ FastStart Essential DNA Green Master, $1 \mu \mathrm{L}$ Forward Primer (10 uM), $1 \mu \mathrm{L}$ Reverse Primer 
(10 uM), and $3 \mu \mathrm{L}$ RNase-Free $\mathrm{ddH}_{2} \mathrm{O}$ water. The PCR was carried out as the following steps:

predegeneration at $95^{\circ} \mathrm{C}$ for $10 \mathrm{~min} ; 45$ cycles of degeneration at $95^{\circ} \mathrm{C}$ for $15 \mathrm{~s}$, annealing at $60^{\circ} \mathrm{C}$ for $15 \mathrm{~s}$, and extension at $72^{\circ} \mathrm{C}$ for $30 \mathrm{~s}$; and melting curve analysis at $65^{\circ} \mathrm{C}-95^{\circ} \mathrm{C}$. RT-qPCR of each cDNA sample was carried out three times as technical replicates. Finally, the relative expression was determined by using the $2^{-\Delta \Delta C t}$ method [28]. The Ct-value (cycle threshold) determined at the end of the reaction indicates the cycle number at which fluorescence passes a fixed threshold. The amplification of $\beta$-Actin was performed as control and reference. The differences between groups were tested by the Paired t-test. $\mathrm{P} \leq 0.05$ and $0.05<\mathrm{P} \leq 0.1$ are considered significant and trend, respectively.

\section{Abbreviations}

T2D: type 2 diabetes; GI: gastrointestinal; PBA: primary bile acids; SBA: secondary bile acid; CA: cholic acid; KOs: Kyoto Encyclopedia of Genes and Genomes (KEGG) orthologs; IBD: inflammatory bowel disease; IGT: impaired glucose tolerance; OGTT: oral glucose tolerance test; LEfSe: linear discriminate analysis (LDA) effect size. PcoA: Principal coordinate analysis; PMA: phorbol 12-myristate 13-acetate; IO: ionomycin; T: T2D individuals before metformin administration who did not have metformin-related GI intolerance after metformin taking; TS: T2D individuals before metformin administration who had metformin-related GI intolerance after metformin taking; Ta: T2D individuals after metformin administration who did not have metformin-related GI intolerance after metformin taking; TSa: T2D individuals after metformin administration who had metformin-related $\mathrm{Gl}$ intolerance after metformin taking.

\section{Declarations}

\section{Ethics approval and consent to participate}

The research activities were approved by the local ethics committee of First People's Hospital of Qujing City. All clinical data collection and genetic diagnoses were performed after obtaining consent from the patients. The objective, materials, and methods of this research and the rights and obligations of the patients have informed patients in oral and written form. Make sure the parents understand all information, then the informed consent was signed before study inclusion. All informed consents were collected and delivered to the study coordinators by the doctor concerned.

\section{Consent for publication}

Not applicable.

\section{Availability of data and materials}


The original sequences were registered in the NCBI SRA database (PRJNA725340). Other data generated or analyzed during this study are included in this article and its additional files.

\section{Competing interests}

The authors declare no conflict of interest.

\section{Funding}

This work was supported by the Applied Basic Research Foundation of Yunnan Province (202101AY070001-050, 2019FE001(-026)); a grant from YNCUB (2019KF003); the National Natural Science Foundation of China (81860255); the scientific research foundation of Yunnan Provincial Department of Education (2018JS159) and Hospital Research Foundation project grant of Qujing N0.1 Hospital of Yunnan Province [2019YJKT09].

\section{Authors' contributions}

Huixiang Zhang and Xue Cao designed the research, analyzed the data, wrote the main manuscript and revised the draft of the manuscript writing. Jiao Lai, Wei Zhang and Weiwen Chen collected the fecal samples and helped to write the manuscript writing. Xun Liu, Limei Wang and Lihuan Zhang collected data from databases and published studies, performed the experiments and prepared figures and tables of this paper. Qilin Gong, Rui Zhao, Dongqing Li, Hehua Huang and Ya Zhao helped to collect the fecal samples and Write the manuscript. Shan Yan, Ming Yu, Xiaodan Liu and Lan-Lan Shi helped to perform the experiments and gave some advice on data analysis.

\section{Acknowledgements}

The grants received from Yunnan Province, the National Natural Science Foundation and Qujing NO.1 Hospital, China are thankfully acknowledged.

\section{Authors' information}

${ }^{1}$ Institute of Neuroscience, Basic Medical College, Kunming Medical University, Kunming 650500, China;

${ }^{2}$ Department of Laboratory Animal Science, Kunming Medical University, Kunming 650500, China;

${ }^{3}$ Department of Endocrinology, Qujing N0.1 Hospital of Yunnan Province, N0.1 Yuanlin Road, Qilin District, Qujing, Yunnan, 655000, China;

${ }^{4}$ Kunming Customs District P.R. China;

${ }^{5}$ Yunnan Key Laboratory of Stem Cell and Regenerative Medicine, Bioengineering Centre, Kunming Medical University, Kunming, P.R. China; 
${ }^{6}$ Department of Anatomy, Histology凶Embryology, Basic Medical College, Kunming Medical University, Kunming 650500, China.

${ }^{7}$ State Key Laboratory for Conservation and Utilization of Bio-Resources in Yunnan『Yunnan University, Kunming 650500, China

\section{References}

1. Bonnet $F$, Scheen A: Understanding and overcoming metformin gastrointestinal intolerance. Diabetes Obes Metab 2017, 19(4):473-481.

2. Forslund K, Hildebrand F, Nielsen T, Falony G, Chatelier EL, Sunagawa S, Prifti E, Vieira-Silva S, Gudmundsdottir V, Pedersen HK et al: Disentangling the effects of type 2 diabetes and metformin on the human gut microbiota. Nature 2015, 528(7581):262-266.

3. Qin J, Li Y, Cai Z, Li S, Zhu J, Zhang F, Liang S, Zhang W, Guan Y, Shen D et al: A metagenome-wide association study of gut microbiota in type 2 diabetes. Nature 2012, 490(7418):55-60.

4. Sun L, Xie C, Wang G, Wu Y, Wu Q, Wang X, Liu J, Deng Y, Xia J, Chen B et al: Gut microbiota and intestinal FXR mediate the clinical benefits of metformin. Nat Med 2018, 24(12):1919-1929.

5. Roy TL, Lécuyer E, Chassaing B, Rhimi M, Lhomme M, Boudebbouze S, Ichou F, Barceló JH, Huby T, Guerin $\mathrm{M}$ et al: The intestinal microbiota regulates host cholesterol homeostasis. BMC Biology 2019, 17:94.

6. Wang K, Liao M, Zhou N, Bao L, Ma K, Zheng Z, Wang Y, Liu C, Wang W, Wang J et al:

Parabacteroides distasonis alleviates obesity and metabolic dysfunctions via production of succinate and secondary bile acids. Cell Rep 2019, 26(1):222-235.

7. Neville BA, Sheridan PO, Harris HMB, Coughlan S, Flint HJ, Duncan SH, Jeffery IB, Claesson MJ, Ross RP, Scott KP et al: Pro-inflammatory flagellin proteins of prevalent motile commensal bacteria are variably abundant in the intestinal microbiome of elderly humans. PLoS One 2013, 8(7):e68919.

8. Costanzo MD, Canani RB: Lactose intolerance: common misunderstandings. Ann Nutr Metab 2018, 73(suppl 4):30-37.

9. Kumar V, Rani A, Goyal L, Dixit AK, Manjaya JG, Dev J, Swamy M: Sucrose and raffinose family oligosaccharides (RFOs) in soybean seeds as influenced by genotype and growing location. $J$ Agric Food Chem 2010, 58(8):5081-5085.

10. Metzakopian E, Lin W, Salmon-Divon M, Dvinge H, Andersson E, Ericson J, Perlmann T, Whitsett JA, Bertone P, Ang S-L: Genome-wide characterization of Foxa2 targets reveals upregulation of floor plate genes and repression of ventrolateral genes in midbrain dopaminergic progenitors. Development 2012, 139(14):2625-2634.

11. Lees CW, Zacharias WJ, Tremelling M, Noble CL, Nimmo ER, Tenesa A, Cornelius J, Torkvist L, Kao J, Farrington $\mathrm{S}$ et al: Analysis of germline GLI1 variation implicates hedgehog signalling in the regulation of intestinal inflammatory pathways. PLoS Med 2008, 5(12):e239. 
12. Shajib MS, Chauhan U, Adeeb S, Chetty Y, Armstrong D, Halder SLS, Marshall JK, Khan WI: Characterization of serotonin signaling components in patients with inflammatory bowel disease. $J$ Can Assoc Gastroenterol 2019, 2(3):132-140.

13. Bednarz-Misa I, Neubauer K, Zacharska E, Kapturkiewicz B, Krzystek-Korpacka M: Whole blood ACTB, B2M and GAPDH expression reflects activity of inflammatory bowel disease, advancement of colorectal cancer, and correlates with circulating inflammatory and angiogenic factors: Relevance for real-time quantitative PCR. Adv Clin Exp Med 2020, 29(5):547-556.

14. Hou Y, Ojo O, Wang L, Wang Q, Jiang Q: A randomized controlled trial to compare the effect of peanuts and almonds on the cardio-metabolic and inflammatory parameters in patients with type 2 diabetes mellitus. Nutrients 2018, 10(11):1565.

15. Tang J, Zeng Z, Wang H, Yang T, Zhang P, Li Y, Zhang A, Fan W, Zhang Y, Yang X et al: An effective method for isolation of DNA from pig faeces and comparison of five different methods. $J$ Microbiol Methods 2008, 75(3):432-436.

16. Ji B, Zhang $X$, Zhang S, Song H, Kong Z: Insights into the bacterial species and communities of a full-scale anaerobic/anoxic/ aerobic wastewater treatment plant by using third-generation sequencing. J Biosci Bioeng 2019, 128(6):744-750.

17. Tedersoo L, Anslan S: Towards PacBio-based pan-eukaryote metabarcoding using full-length ITS sequences. Env Microbiol Rep 2019, 11(5):659-668.

18. Edgar RC, Haas BJ, Clemente JC, Quince C, Knight R: UCHIME improves sensitivity and speed of chimera detection. Bioinformatics 2011, 27 (16):2194-2200.

19. Haas BJ, Gevers D, Earl AM, Feldgarden M, Ward DV, Giannoukos G, Ciulla D, Tabbaa D, Highlander SK, Sodergren E et al: Chimeric 16S rRNA sequence formation and detection in Sanger and 454pyrosequenced PCR amplicons. Genome research 2011, 21(3):494-504.

20. Edgar RC: UPARSE: highly accurate OTU sequences from microbial amplicon reads. Nat Methods 2013, 10(10):996-998.

21. Wang Q, Garrity GM, Tiedje JM, Cole JR: Naive Bayesian classifier for rapid assignment of rRNA sequences into the new bacterial taxonomy. App/ Environ Microbiol 2007, 73(16):5261-5267.

22. Quast C, Pruesse E, Yilmaz P, Gerken J, Schweer T, Yarza P, Peplies J, Glöckner FO: The SILVA ribosomal RNA gene database project: improved data processing and web-based tools. Nucleic Acids Res 2013, 41(D1):D590-D596.

23. Douglas GM, Maffei VJ, Zaneveld J, Yurgel SN, Brown JR, Taylor CM, Huttenhower C, Langille MGI: PICRUSt2: An improved and extensible approach for metagenome inference. bioRxiv [preprint] 2019.

24. García-Calzón S, Perfilyev A, Martinell M, Ustinova M, Kalamajski S, Franks PW, Bacos K, Elbere I, Pihlajamäki J, Volkov $P$ et al: Epigenetic markers associated with metformin response and intolerance in drug-naïve patients with type 2 diabetes. Sci Trans/ Med 2020, 12(561):eaaz1803.

25. Szklarczyk D, Gable AL, Lyon D, Junge A, Wyder S, Huerta-Cepas J, Simonovic M, Doncheva NT, Morris JH, Bork P et al: STRING v11: protein-protein association networks with increased coverage, 
supporting functional discovery in genome-wide experimental datasets. Nucleic Acids Res 2019, 47(D1):D607-D613.

26. Shannon P, Markiel A, Ozier O, Baliga NS, Wang JT, Ramage D, Amin N, Schwikowski B, Ideker T: Cytoscape: a software environment for integrated models of biomolecular interaction networks. Genome Res 2003, 13(11):2498-2504.

27. Xie H, Li B, Chang Y, Hou X, Zhang Y, Guo S, Miao Y, Wang Q, Chen S, Su Y et al: Selection and validation of reference genes for RT-qPCR analysis in Spinacia oleracea under abiotic stress. Biomed Res Int 2021, 2021:4853632.

28. Schmittgen TD, Livak KJ: Analyzing real-time PCR data by the comparative $\mathrm{C}(\mathrm{T})$ method. Nat Protoc 2008, 3(6):1101-1108.

\section{Figures}


$\mathrm{a}$

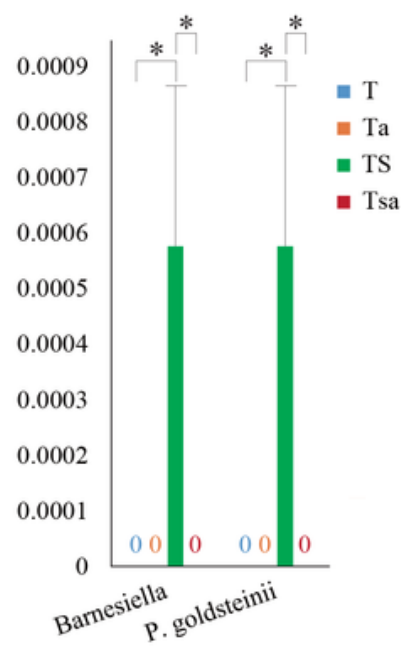

b

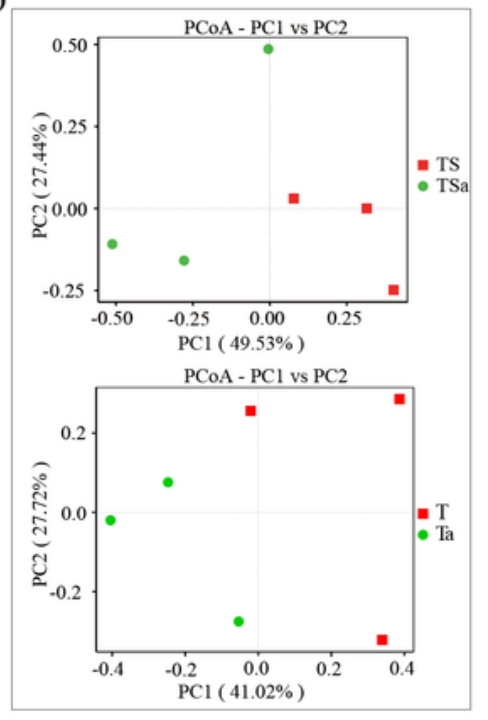

d
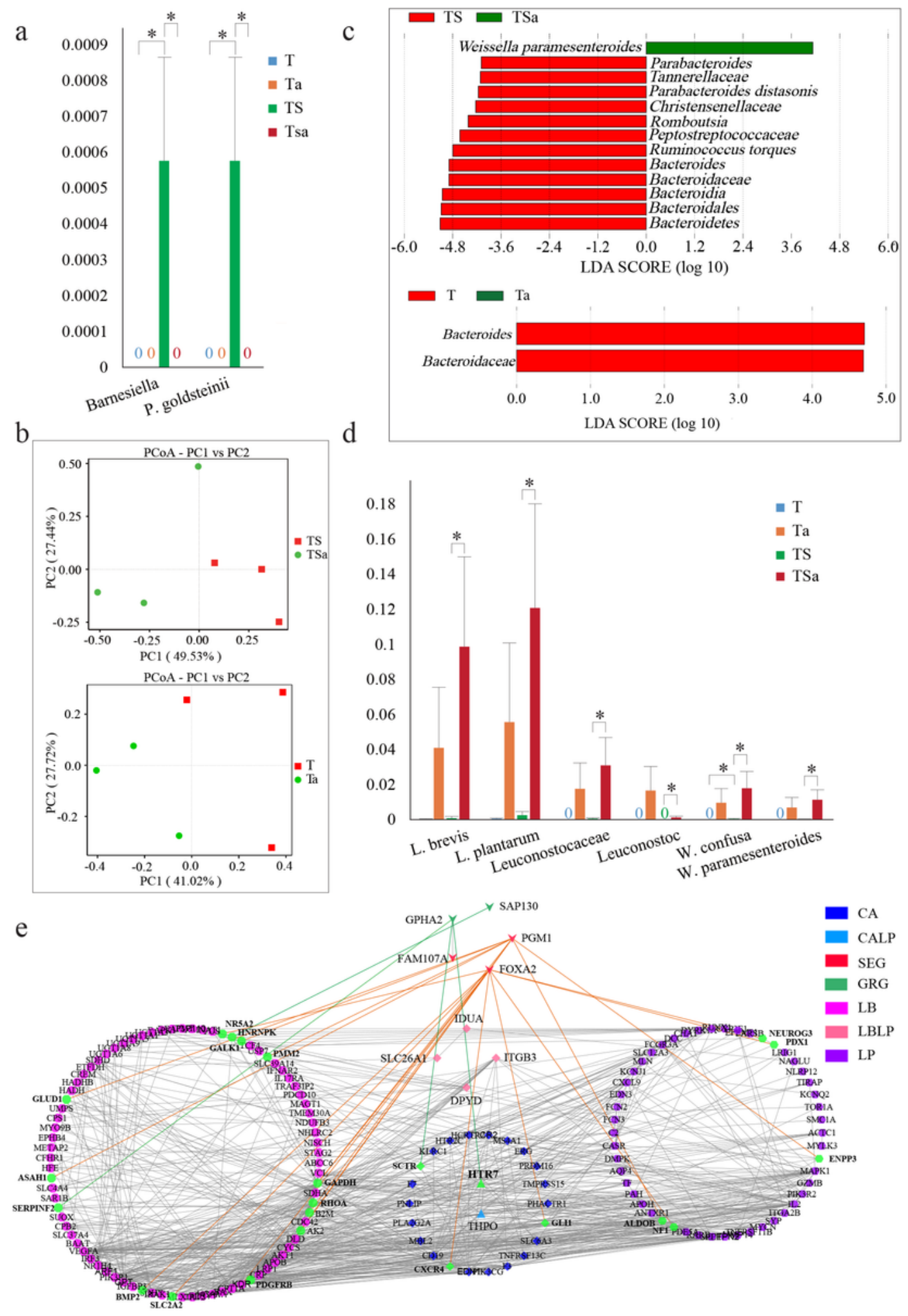

Figure 1

Comparison of intestinal flora before and after taking metformin between people who had intestinal side effects and those without intestinal side effects after taking metformin, and the interaction analysis of the differential flora and side effects related genes. a,d. Relative abundance of differential intestinal flora among four groups. b,c. Principal coordinate analysis (PCoA) and linear discriminant analysis (LDA) effect size (LEfSe) analysis among groups. The red represents before taking metformin, and the green 
represents after taking metformin. e. Interaction analysis of the glycemic response and intolerancerelated genes reported in the literature, differential intestinal flora, and Cholic acid. CA Cholic acid, CALP Cholic acid and Lactobacillus plantarum, SEG side effects related genes, GRG glycemic response-related genes, LB Lactobacillus brevis, LBLP Lactobacillus brevis, and Lactobacillus plantarum.

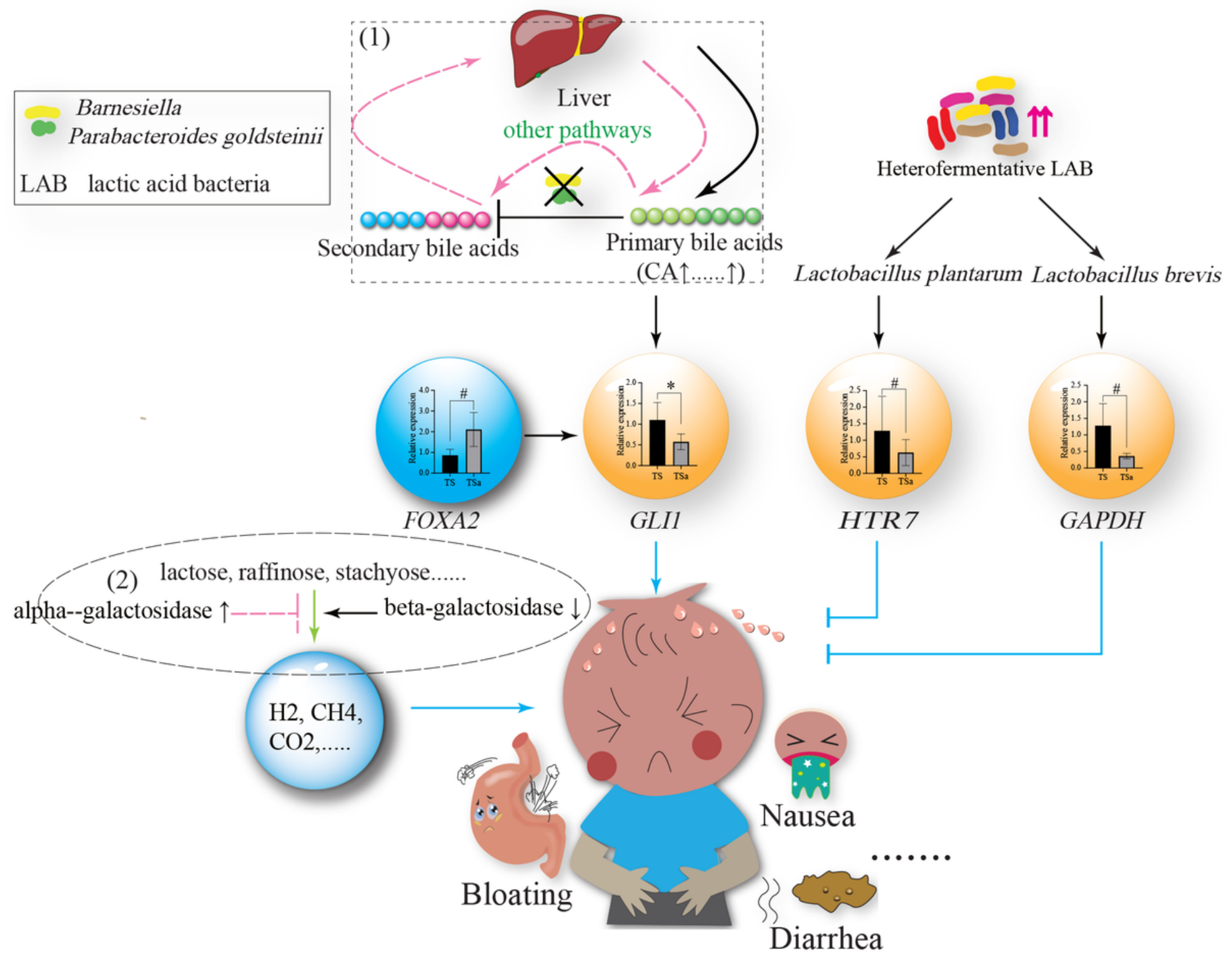

Figure 2

Diagram of the possible gut microbial mechanism of intestinal side effects caused by metformin. The black arrow indicates the result of the GEO analysis and RT-qPCR test, and the blue arrow indicates the results from the literature analysis.

\section{Supplementary Files}

This is a list of supplementary files associated with this preprint. Click to download.

- FigureS1.pdf 
- FigureS2.pdf

- Figures3.pdf

- SupplementaryFigureLegend.docx

- SupplementaryTables.xlsx

- SupplementaryEthicalPermission.pdf

- SupplementaryInformedConsentTemplate.pdf 